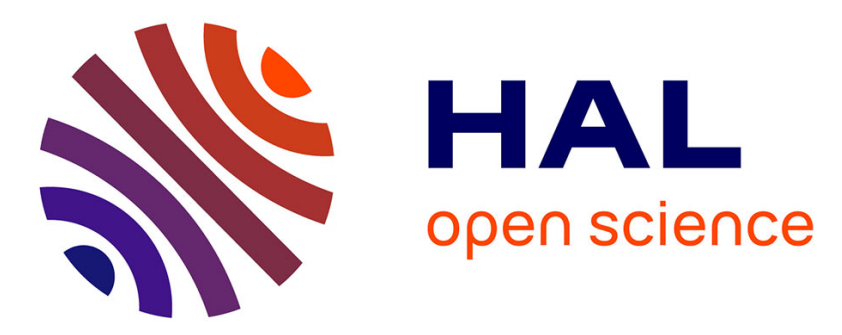

\title{
Détection d'endommagement dans les composites fibres/résine à l'aide de la technologie micro-onde
}

\author{
Jérome Rossignol, Alain Thionnet
}

\section{To cite this version:}

Jérome Rossignol, Alain Thionnet. Détection d'endommagement dans les composites fibres/résine à l'aide de la technologie micro-onde. Revue des composites et des matériaux avancés = Journal of Composite and Advanced Materials, 2006, 16, pp.263-278. hal-00143717

\section{HAL Id: hal-00143717 https://hal.science/hal-00143717}

Submitted on 26 Apr 2007

HAL is a multi-disciplinary open access archive for the deposit and dissemination of scientific research documents, whether they are published or not. The documents may come from teaching and research institutions in France or abroad, or from public or private research centers.
L'archive ouverte pluridisciplinaire HAL, est destinée au dépôt et à la diffusion de documents scientifiques de niveau recherche, publiés ou non, émanant des établissements d'enseignement et de recherche français ou étrangers, des laboratoires publics ou privés. 


\title{
Détection d'endommagement dans les composites fibres/résine à l'aide de la technologie micro-onde
}

\author{
Jérôme Rossignol *,** _ Alain Thionnet *, *** \\ * Université de Bourgogne, Mirande, BP 47870, F-21078 Dijon \\ ** Laboratoire de Réactivité des Solides, CNRS UMR 5613 \\ *** Centre des Matériaux Mines Paris, Paristech, CNRS UMR 7633 \\ BP 87, F-91003 Evry cedex \\ jerome.rossignol@u-bourgogne.fr, alain.thionnet@ensmp.fr
}

\begin{abstract}
RÉSUMÉ. On présente dans cet article une méthode novatrice de contrôle non destructif d'endommagement dans les matériaux composites. Elle est fondée sur l'observation et l'analyse des modifications des propriétés diélectriques d'un milieu dues à un endommagement. Plus précisément, son originalité réside dans le fait que le diagnostic cherché est obtenu via un circuit résonant microbande soumis à un champ électromagnétique dans la gamme des micro-ondes. Dans cet article on montre la faisabilité de la méthode sur la détection de la rupture des fibres d'une éprouvette en composite unidirectionnel soumise à un effort de flexion et on indique en quoi elle possède potentiellement des atouts pour être une alternative intéressante aux méthodes existantes.

ABSTRACT. This paper presents a original technique to detect the damage in composite materials. This novel non destructive method is based on the observation and the analysis of the variations of the dielectric properties of the material due to the damage phenomenon. This originality comes from the use of a passive resonator in a microstrip structure in the microwave frequency. This method is here apply in the case of the detection of fibre breakings susceptible to appear in an unidirectionnal composite submitted to flexural loading.

MOTS-CLÉS : détection d'endommagement, composites, caractérisation diélectrique, microondes, méthodes expérimentales.

KEYWORDS: damage detection, composites, dielectric characterization, microwaves, experimental techniques.
\end{abstract}

Revue des composites et des matériaux avancés. Volume 16 - n 3/2006, pages 263 à 278 


\section{Introduction et but de l'étude}

En particulier dans le domaine des transports (ferroviaires, automobiles, aéronautiques), l'accroissement de l'utilisation des matériaux composites et le renforcement des normes de sécurité conduisent à l'obligation d'un contrôle périodique accru des structures. Ce contrôle a notamment pour objectif d'en prévenir toute défaillance due à une perte de ses caractéristiques mécaniques induites par de l'endommagement.

La littérature met en évidence de nombreuses méthodes possibles de contrôle non destructif de l'endommagement au sein des matériaux et des structures. Cependant, le cadre de nos exigences, dans lequel on inscrit les notions de coût, de rapidité d'une mesure réalisée obligatoirement in situ (afin de réduire les coûts d'immobilisation) et d'adaptation aux composites, fait que le nombre des techniques envisageables est finalement assez réduit. Ainsi, les méthodes fondées sur le principe de la thermographie infrarouge, de l'émission acoustique, de la méthode EMIR (ElectroMagnetic InfraRed) et de l'imagerie micro-onde conduisent, en général, assez aisément à une expertise adaptée à notre demande.

Aucune méthode n'est cependant idéale et chacune possède ses propres avantages et ses propres inconvénients. La question qu'il est alors légitime de poser est la suivante : quelles propriétés doit posséder la méthode idéale pour répondre à nos exigences, c'est-à-dire permettre l'expertise fiable, aisée, in situ et non destructive, de l'intégrité face à l'endommagement, d'une structure composite ? Pour répondre au mieux à nos attentes, en plus d'assurer une mesure in situ aisée, la méthode doit permettre :

- de distinguer la nature d'endommagements différents susceptibles d'être simultanément présents ;

- de quantifier les différents endommagements;

- de localiser les endommagements présents, notamment dans la profondeur du matériau.

Le but de notre étude est de proposer une technique novatrice de contrôle non destructif des endommagements dans les composites, alternative et complémentaire des méthodes existantes, respectant nos exigences. Cette méthode est fondée sur l'observation et l'analyse des modifications des propriétés diélectriques d'un milieu dues à un endommagement. Plus précisément, son originalité réside dans le fait que le diagnostic cherché est obtenu au moyen de la réponse, dans la gamme des micro-ondes, d'un circuit passif de type microruban ayant une fonction résonante. Dans cet article, on montre la faisabilité de la méthode en mettant en évidence sa capacité à détecter l'anisotropie du milieu ainsi que les ruptures de fibres au sein d'une éprouvette en composite unidirectionnel soumise à un effort de flexion. Egalement, on indique ses atouts potentiels par rapport aux méthodes existantes. En revanche, on ne répond pas pour l'instant ni à la question de savoir si la méthode proposée est capable de quantifier et de qualifier un endommagement, ni au calcul explicite des propriétés diélectriques d'un milieu équivalent à celui endommagé, dont on ne donne que le principe. 


\section{Synthèse bibliographique}

Les techniques fondées sur la technologie des rayonnements X ((Schilling et al., 2005)...), les fibres optiques ((Wevers et al., 2006), (Pevzner et al., 2005), (Xu et al., 2005), (DeOliveira et al., 2004), (Okabe et al., 2002)...), les ultrasons ((Scarponi et al., 2000), (Aymerich et al., 2000)...), les mesures de courant ((Abry et al., 2001), (Schueler et al., 2001)...) sont des techniques évidemment tout à fait envisageables pour détecter les endommagements au sein des composites. Toutefois, leur mise en œuvre lourde fait que, dans le cadre de nos exigences, on leur préfère des méthodes qui utilisent une infrastructure de fonctionnement plus légère.

Les méthodes basées sur la thermographie infrarouge ((Ball et al., 1998), (Mian et al., 2004)...) consistent à exciter un matériau par un apport d'énergie (mécanique, photonique, chauffage par induction, lampes, flash...) et à mesurer, à l'aide d'une caméra infrarouge, l'élévation de température résultante. Ces méthodes sont déjà appliquées aux composites et renseignent dans une certaine mesure sur la nature du défaut, surtout si sa géométrie est caractéristique (délaminage par exemple).

Les méthodes basées sur les principes de l'émission acoustique ((Surgeon et al., 1999), (Rippert et al., 2000), (Hill et al., 1998)... ) consistent à recueillir et à analyser les ondes acoustiques émises par le matériau lui-même. Ces ondes sont créées par les microdéplacements internes induits dans notre cas par les phénomènes d'endommagement au sein du composite (décohésion fibre/matrice, fissuration intralaminaire, rupture de fibres, délaminage...). Ces méthodes sont déjà utilisées en exploitation industrielle pour le contrôle des réservoirs de bus en composite unidirectionnel ((Blassiau, 2005), (Blassiau et al., 2006a), (Blassiau et al., 2006b)) pour lesquels le seul endommagement très majoritairement présent, compte tenu de l'orientation des fibres et des sollicitations, est la rupture de fibres. Ce dernier point est une remarque importante. En effet, malgré les nombreux travaux existants et en cours, il n'est pas encore parfaitement démontré que cette méthode puisse distinguer un type d'endommagement d'un autre dans le cas où plusieurs dégradations sont simultanément présentes dans le matériau (la fissuration intralaminaire et la rupture de fibre, par exemple). Il n'est pas non plus parfaitement démontré que cette méthode localise finement les dégradations, notamment dans l'épaisseur du matériau composite.

La méthode EMIR ((Balageas et al., 1998), (Pliquet et al., 1999)...) utilise quant à elle une antenne ou un guide d'ondes qui émet un champ électromagnétique microonde sur la structure à tester. Le passage de l'onde électromagnétique à travers le matériau modifie sa répartition. Un film absorbant mince préalablement collé sur la face opposée à celle vue par l'antenne, transforme l'énergie électromagnétique en chaleur. Cet échauffement est alors détecté par une caméra infrarouge. Pour cette méthode, l'interprétation des images enregistrées peut présenter l'inconvénient d'une expertise difficile dans l'épaisseur du milieu.

On peut également citer des techniques qui utilisent l'interférométrie laser ((Minnaar et al., 2004)...) ou des capteurs piézo-électriques ((Yan et al., 2002)...). 
Enfin, deux grandes familles de méthodes utilisent des ondes électromagnétiques dans le domaine des micro-ondes. La distinction entre elles deux réside dans le fait que l'une agit avec contact et l'autre sans contact. Les méthodes sans contact disposent de deux antennes, l'une émettrice l'autre réceptrice d'un champ électromagnétique micro-onde. Le milieu à expertiser est placé entre les deux antennes. Le champ émis traverse le milieu et l'onde transmise est récupérée et analysée par l'antenne émettrice ((Sandacci et al., 2005)...). Les méthodes avec contact les plus fréquemment utilisées placent le milieu à expertiser soit à l'extrémité d'un guide d'onde, soit dans une structure propagative coaxiale dans lequel il joue le rôle de l'isolant. Un analyseur de réseau vectoriel caractérise alors le matériau notamment en analysant la réflexion et la transmission d'ondes électromagnétiques micro-ondes à travers le composant ((Shrestha et al., 2005), (Akuthota et al., 2004), (Hughes et al., 2002), (Kagawa et al., 2001), (Ganchev et al., 1995), (Huber et al., 1996), (LeGallou et al., 2002)...). Dans les deux cas, ces techniques ne permettent pas aisément, voire pas du tout, la mise en place de l'expertise d'une structure volumineuse avec des formes complexes.

\section{Description de la méthode}

Si c'est l'infrastructure même des méthodes précédentes qui nous conduit à les abandonner, en revanche, la technique des micro-ondes est celle qui nous intéresse dès lors qu'elle est utilisée sous une autre forme. En effet, dans le domaine des télécommunications aux fréquences micro-ondes, les lignes de transmission (hors espace libre) sont généralement soit des guides d'ondes soit des circuits microrubans. Les grandeurs caractéristiques de ces circuits sont influencées notamment par les propriétés diélectriques du matériau qui constitue l'un de leurs éléments constitutifs, appelé le substrat. L'idée de la méthode de détection d'endommagement que l'on propose est la suivante : utiliser comme matériau du substrat du circuit, le matériau dont on veut identifier l'état d'endommagement. En s'endommageant, les propriétés diélectriques du matériau-substrat vont se modifier. Par voie de conséquence, les valeurs des grandeurs caractéristiques du circuit vont également varier et seront donc révélatrices de l'état d'endommagement du matériau-substrat.

\subsection{Circuit microruban : principe, mesures et calculs des grandeurs caractéristiques}

S'ils peuvent évidemment se propager dans l'espace, les champs électromagnétiques sont propagés le plus couramment dans des guides d'ondes, des lignes bifilaires et des circuits microrubans. Un circuit microruban (figure 1) se compose d'un substrat dont l'une des faces est métallisée (par un film de cuivre collé dans notre cas), jouant le rôle de plan de masse. L'autre n'est recouverte que partiellement de métal sous la forme d'un motif conducteur : c'est le ruban. La forme du motif, aux gammes de fréquences utilisées, confère au microruban une fonction électronique de base, soit de filtre soit de résonateur. Cette structure constitue une ligne de propagation pour 
une onde électromagnétique. L'étude de la propagation d'un champ électromagnétique dans un circuit microruban est rendue difficile en raison d'une propagation qui s'effectue simultanément dans le substrat, mais également dans l'air. Cependant, des hypothèses légitimes en première approximation sont couramment faites :

- les modes de propagation des champs sont à dominante de type Transverse ElectroMagnetic (TEM);

- les milieux testés sont à faibles pertes diélectriques.

On adopte ces hypothèses dans le cadre de cette étude. Dans ces conditions, on peut définir pour la structure de propagation concernée une impédance dite caractéristique et notée $Z_{c}$. Dans le cas où le circuit est résonant, il est caractérisé par une fréquence de résonance, notée $f_{c}$, ainsi qu'un facteur de qualité $Q$. Pour arriver au résultat attendu ici, les grandeurs $Z_{c}$ et $Q$ ne sont pas les plus importantes : elles ne sont donc plus évoquées dans la suite.

L'accès expérimental à $f_{c}$ se fait de manière indirecte. Plus précisément, on utilise un analyseur de réseaux vectoriel sur le plan fréquentiel relié par un câble coaxial (d'impédance $Z_{\text {coaxial }}$ ) à l'une des extrémités du ruban, l'autre extrémité du ruban étant reliée à une impédance $Z_{\text {charge }}$ de valeur donnée et fixe, ici égale à $Z_{\text {coaxial }}$. On définit le plan de référence des mesures à l'entrée du ruban. Dans ce plan, l'analyseur mesure l'onde électromagnétique incidente qu'il émet. Il y mesure également l'onde réfléchie pour laquelle on associe, pour ce plan, un coefficient de réflexion complexe appelé usuellement $S_{11}$ et noté ici $\Gamma$. L'examen des mesures du coefficient $\Gamma$ en fonction de la fréquence permet d'identifier $f_{c}$.

Dans le cadre des hypothèses faites et qui permettent de simplifier le problème de la propagation de l'onde dans le circuit, il est possible de calculer $\Gamma$ et $f_{c}$. Pour des géométries simples, des formules analytiques sont accessibles et basées sur le modèle de Hammerstadt (Hammerstad, 1975). Le principe de ce modèle est le suivant : le circuit microruban est rendu équivalent à un circuit où le ruban du circuit original est entouré par un milieu homogène équivalent dont la permittivité relative, dite efficace, est notée $\epsilon_{r}^{e f f}$. Cette permittivité se calcule analytiquement et dépend explicitement de la géométrie du substrat, du plan de masse et du ruban, ainsi que de la permittivité diélectrique relative du substrat, $\epsilon_{r}$. Pour des géométries complexes, il convient d'avoir recours à des techniques numériques qui résolvent les équations de Maxwell (par exemple, différences finis, éléments finis, méthodes des moments).

Les grandeurs $\Gamma$ et $f_{c}$ dépendent de la géométrie du ruban et du substrat. Elles dépendent aussi des propriétés physiques des matériaux qui les constituent, notamment de la permittivité diélectrique relative $\epsilon_{r}$ du matériau constitutif du substrat. Toutes ces grandeurs étant fixées, en première approximation, $f_{c}$ et $\epsilon_{r}$ ne dépendent plus que de la fréquence de l'onde qui parcours le circuit. En ce qui concerne $\epsilon_{r}$, cette dépendance est très faible pour les milieux que l'on étudie ici. On suppose donc implicitement admis dans la suite que la permittivité des milieux utilisés comme substrat est indépendante de la fréquence. 


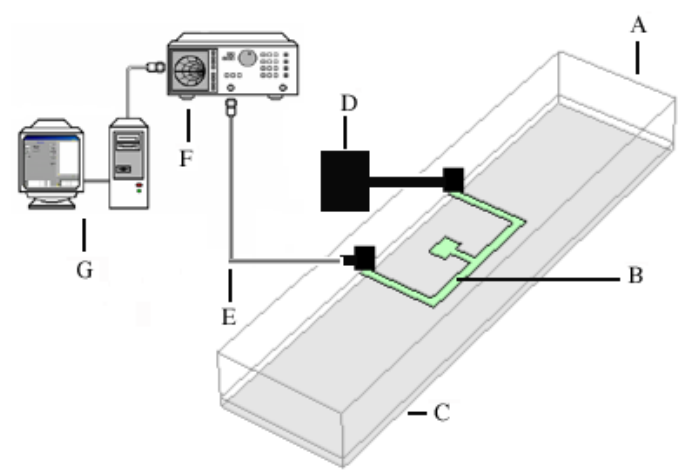

Figure 1. Schéma de principe d'un circuit microruban (A : substrat, $B$ : ruban, $C$ : plan de masse, $D$ : charge $Z_{\text {charge, }} E$ : câble coaxial, $F$ : analyseur de réseaux, $G$ : système d'acquisition des mesures)

\subsection{Principe de la méthode}

Notre objectif est de détecter un endommagement au sein d'un matériau composite. Dans le cadre de la technique présentée, le rôle du substrat est joué par le matériau à expertiser et le diagnostic cherché est basé sur le postulat suivant : en l'absence de tout phénomène dissipatif excepté l'endommagement que l'on souhaite détecter, en supposant constantes les caractéristiques géométriques des différents éléments du circuit ainsi que les propriétés physiques du matériau constitutif du plan de masse et du ruban, si l'on relève des variations sur les valeurs de $\Gamma$ et $f_{c}$, alors ces variations seront imputées à des variations des propriétés diélectriques du matériaususbtrat, révélatrices de l'endommagement au sein de ce matériau. Pour cela, on se place sous l'Hypothèse des Petites Perturbations qui justifie le fait que les variations de dimensions du ruban sont suffisamment petites pour qu'elles ne modifient pas les caractéristiques du circuit, et on estime qu'aucun autre phénomène dissipatif notable, susceptible de modifier les propriétés diélectriques et mécaniques du milieu analysé, autre que l'endommagement, n'est présent. Ces hypothèses sont évidemment mises en défaut au voisinage de l'état de rupture.

Dans le cadre de la méthode proposée, on mesure d'abord $\Gamma$ et $f_{c}$ à différents instants choisis. Puis, pour chaque point de mesure, on en déduit $\epsilon_{r}$, la permittivité relative du matériau-substrat équivalent à celui endommagé, en utilisant un modèle (analytique ou numérique) dans un processus inverse : on ajuste la valeur de $\epsilon_{r}$ jusqu'à ce que les valeurs calculées de $\Gamma$ et $f_{c}$ coïncident avec les valeurs mesurées. 


\section{Détection de l'anisotropie d'un composite unidirectionnel}

Le premier test de mise en évidence de la faisabilité de la méthode consiste à détecter l'anisotropie d'un composite unidirectionnel vierge d'endommagement. Pour cela on utilise deux circuits microrubans résonants dont la géométrie et le substrat sont identiques : une éprouvette carrée de composite unidirectionnel verre/époxy, de côté $50 \mathrm{~mm}$ et d'épaisseur $4 \mathrm{~mm}$. Leur différence repose sur la mise en place du ruban : pour l'un, il est aligné avec les fibres du composite (circuit "type $0^{\circ}$ "), pour l'autre, il est placé perpendiculairement (circuit "type $90^{\circ}$ ").

On mesure un coefficient de réflexion différent entre les deux circuits et, par suite, on met en évidence une fréquence de résonance différente pour les deux circuits (figure 2). Les mesures sont effectuées à $2 \mathrm{GHz}$. La méthode détecte donc bien l'anisotropie du milieu. Des mesures identiques utilisant des micro-ondes propagées dans des guides ouverts ont été faites (Bleay et al., 1999) sur des matériaux similaires. Elles confirment le caractère anisotrope des propriétés diélectriques de ces milieux.

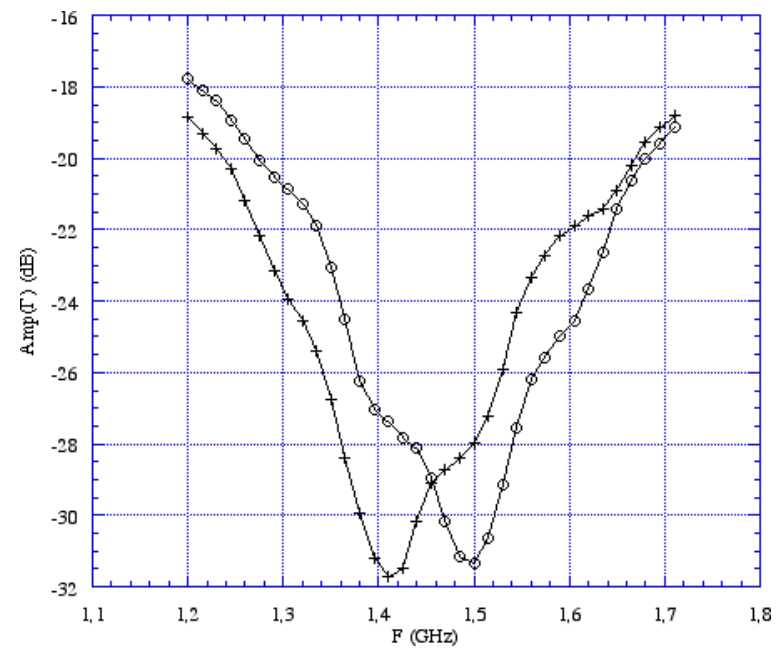

Figure 2. Amplitude de $\Gamma(\operatorname{Amp}(\Gamma)$, unité : $d B)$ en fonction de la fréquence $(F$, unité : $\mathrm{GHz})$ o : circuit "type $0^{\circ} ",+$ : circuit "type $90^{\circ} "$

\section{Détection des ruptures de fibres au sein d'un composite unidirectionnel en flexion}

Le second test de mise en évidence de la faisabilité de la méthode consiste à détecter les ruptures de fibres au sein d'un composite unidirectionnel sollicité. La méthodologie de la campagne expérimentale utilisée est la suivante : 
- un essai de flexion sur une éprouvette unidirectionnelle à $0^{\circ}$ permet de contrôler l'apparition des ruptures de fibres et de détecter, en exploitant la courbe force/flèche, une rupture de pente révélatrice de l'initiation de cet endommagement;

- cette éprouvette est le substrat d'un circuit microruban résonant pour lequel on mesure au cours de l'essai, la grandeur $\Gamma$.

\subsection{Description de l'essai de flexion 3 points : éprouvette, sollicitation, instrumentation, circuit et principe des mesures}

Le repère du laboratoire est le repère orthonormé $R=(O, \vec{x}, \vec{y}, \vec{z})$ (figure 3 ), sa base est notée $B$. L'éprouvette est en matériau composite unidirectionnel verre/époxy (dont les propriétés mécaniques et à rupture sont connues). La base du repère d'anisotropie du matériau est la base $B_{m a t}=\left(\vec{X}_{1}, \vec{X}_{2}, \vec{X}_{3}\right)$ : l'axe orienté par le vecteur $\vec{X}_{1}$ désigne l'axe des fibres, les fibres sont contenues dans le plan $\left(\vec{X}_{1}, \vec{X}_{2}\right)$. La base du repère associé à l'éprouvette est la base $B_{\text {spe }}=\left(\overrightarrow{e_{1}}, \overrightarrow{e_{2}}, \overrightarrow{e_{3}}\right)$ (figure 4 ). Pour les essais réalisés, les bases $B, B_{\text {mat }}$ et $B_{\text {spe }}$ coïncident. La longueur de l'éprouvette, indiquée par le vecteur $\overrightarrow{e_{1}}$, est de $150 \mathrm{~mm}$, sa largeur, indiquée par le vecteur $\overrightarrow{e_{2}}$, est de $48.5 \mathrm{~mm}$ et son épaisseur, indiquée par le vecteur $\overrightarrow{e_{3}}$, est de $4,5 \mathrm{~mm}$. Elle repose sur 2 appuis parallèles cylindriques dont les axes sont indiqués par le vecteur $\vec{y}$. Leur diamètre est de $10 \mathrm{~mm}$, leur longueur de $50 \mathrm{~mm}$ et ils sont distants de $100 \mathrm{~mm}$. Un couteau cylindrique (de $10 \mathrm{~mm}$ de diamètre et $50 \mathrm{~mm}$ de longueur), parallèle aux appuis, applique une sollicitation verticale descendante $-F(t) \vec{z}$ sur la peau supérieure de l'éprouvette. Le déplacement du couteau est noté $-D(t) \vec{z}$.

Si les mesures des grandeurs caractéristiques d'un circuit peuvent être faites a priori à n'importe quelle fréquence, en revanche, s'il est résonant, celles faites au voisinage de sa fréquence de résonance $f_{c}$ permettent d'amplifier notablement d'éventuelles variations. Plus précisément, dans notre cas, l'intérêt de choisir un circuit résonant réside dans le fait que toute variation de la permittivité du substrat due à l'endommagement, détectée à la fréquence de résonance, sera amplifiée sur la réponse du circuit. Aussi, les dimensions du ruban ont été calculées (à l'aide du modèle de Hammerstadt et en se donnant un ordre de grandeur de la permittivité du matériau, trouvé dans la littérature) de manière à ce que le circuit soit résonant et que sa fréquence de résonance $f_{c}$ soit voisine du centre de la gamme fréquentielle de l'analyseur utilisé, soit $1.7 \mathrm{GHz}$. Des mesures faites sur éprouvette non chargée permettent de vérifier que les calculs effectués sont corrects.

Finalement, le ruban conducteur (en cuivre) du circuit a la forme d'un U (figure 4). Il est collé sur la peau inférieure de l'éprouvette-substrat. La peau sollicitée étant la peau supérieure, il ne sera ainsi pas endommagé par le couteau. Sa branche inférieure, centrée dans la largeur et la longueur de l'échantillon, est alignée avec les fibres. Les branches verticales arrivent jusqu'à l'un des bords latéraux : l'une est reliée à l'impédance $Z_{\text {charge }}$, l'autre au câble coaxial relié à l'analyseur de réseaux. Le plan de masse (en cuivre, 30 microns d'épaisseur) est collé sur la peau supérieure de l'éprouvette : il est donc en contact avec le couteau. 
Un premier lot d'essais a permis de calibrer et de mettre au point la machine de flexion et l'ensemble du dispositif de mesure. Ensuite, deux essais ont été réalisés et donnent des résultats identiques. Les essais sont pilotés en déplacement à une vitesse constante de $2 \mathrm{~mm} / \mathrm{mn}$. Deux types de profil de déplacement du couteau en fonction du temps ont été utilisés :

- pour les essais de calibration, application d'un déplacement allant de la valeur nulle jusqu'à une valeur provoquant la rupture de l'éprouvette ;

- pour les deux essais suivants, application d'un profil de déplacement de type charge/décharge, la valeur de la charge étant augmentée successivement jusqu'à atteindre la rupture, la décharge ramenant toujours le déplacement à une valeur nulle (figure 5).

A l'aide d'un capteur d'effort, on mesure la force appliquée au couteau. On accède ainsi à la courbe force mesurée $(\mathrm{F}$, unité : $\mathrm{N})$ en fonction du déplacement imposé $(\mathrm{D}$, unité : mm) (figure 6).

A l'aide de l'analyseur de réseau, on mesure l'amplitude, les parties réelles et imaginaires du coefficient de réflexion $\Gamma$ aux valeurs maximales du déplacement atteint lors des créneaux (figure 5, points $\left(B_{i=1, \ldots, 5}\right)$ ) ainsi qu'aux retours à une valeur nulle (figure 5 , points $\left(A_{i=1, \ldots, 5}\right)$ ). En chacun de ces points, l'essai est arrêté quelques secondes afin d'effectuer les mesures pour un balayage fréquenciel allant de $300 \mathrm{kHz}$ à $3 \mathrm{GHz}$ par pas de $15 \mathrm{MHz}$.

Il est à noter que le collage du ruban ainsi que les soudures des connectiques ont été réalisés avec le plus grand soin afin de pallier à tout défaut de décollement susceptible de fausser les mesures. De même, à chaque mesure, la surface de l'éprouvette a été expertisée (visuellement) en vue de vérifier si l'intégrité du collage des parties cuivrées était préservée. Dans le cas contraire (proche de la rupture de l'éprouvette), les mesures n'ont pas été comptabilisées. Les résultats des mesures micro-ondes présentés ici satisfont à ces conditions. Toutefois, il convient de préciser qu'en substituant le film continu du plan de masse par une grille fine, on n'a observé expérimentalement aucune différence significative des résultats de mesure : de légères coupures dans le plan de masse ne perturbent finalement pas les résultats de mesures.

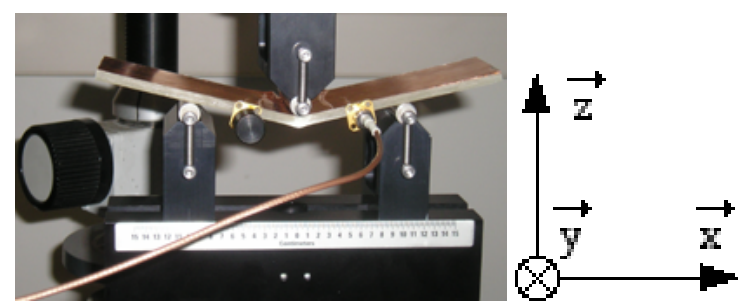

Figure 3. Dispositif de flexion 


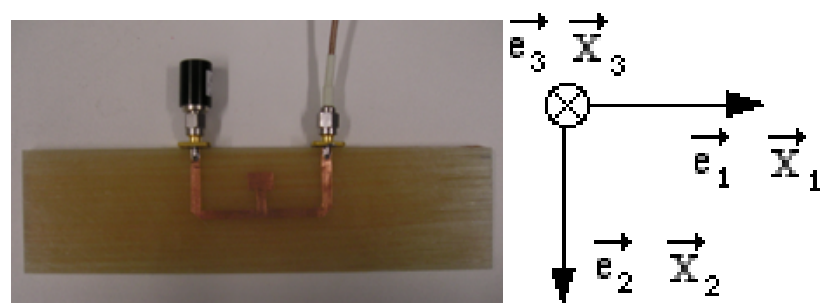

Figure 4. Instrumentation de l'éprouvette (vue de la peau inférieure)

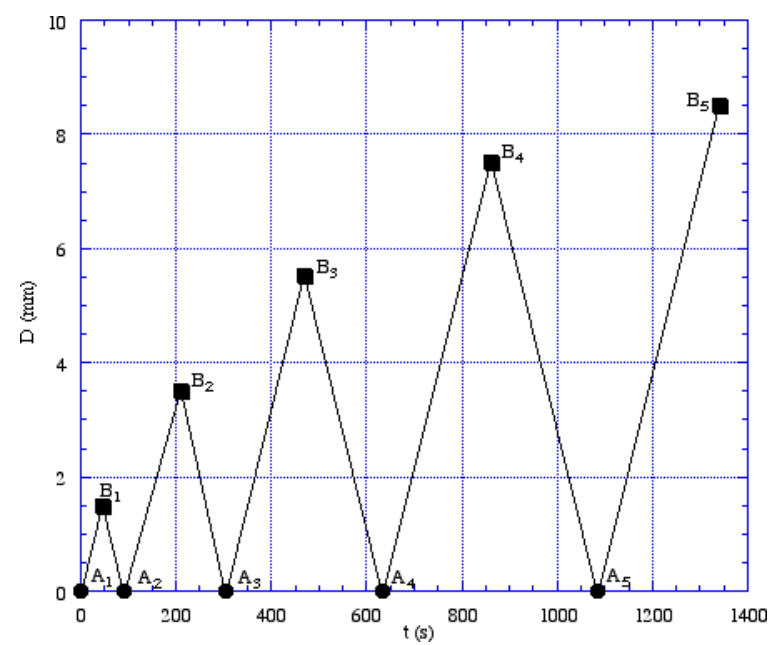

Figure 5. Déplacement appliqué ( $D$, unité : $m m$ ) en fonction du temps ( $t$, unité : s)

\subsection{Analyse des mesures mécaniques}

Afin d'analyser l'expérience en termes de grandeurs mécaniques, on la modélise par la méthode des éléments finis. L'objectif de ce calcul n'étant que d'obtenir un ordre de grandeur de certaines quantités (flèche, critère de rupture de fibres, notamment), la modélisation est donc simple. Notamment, les conditions aux limites qui imposent que les nœuds du maillage en contact avec les appuis ont un déplacement vertical nul, induisent que cette modélisation ne tient pas compte du léger glissement vers le bas des points de contact entre l'éprouvette et les appuis. La discrétisation de l'éprouvette (dans laquelle est pris en compte le plan de masse en cuivre) est réalisée à l'aide d'éléments quadratiques à 20 nœuds (C3D20). Le maillage de l'éprouvette est découpé régulièrement suivant l'axe des appuis. Le comportement du matériau composite est supposé élastique linéaire orthotrope et un critère local simple de rup- 


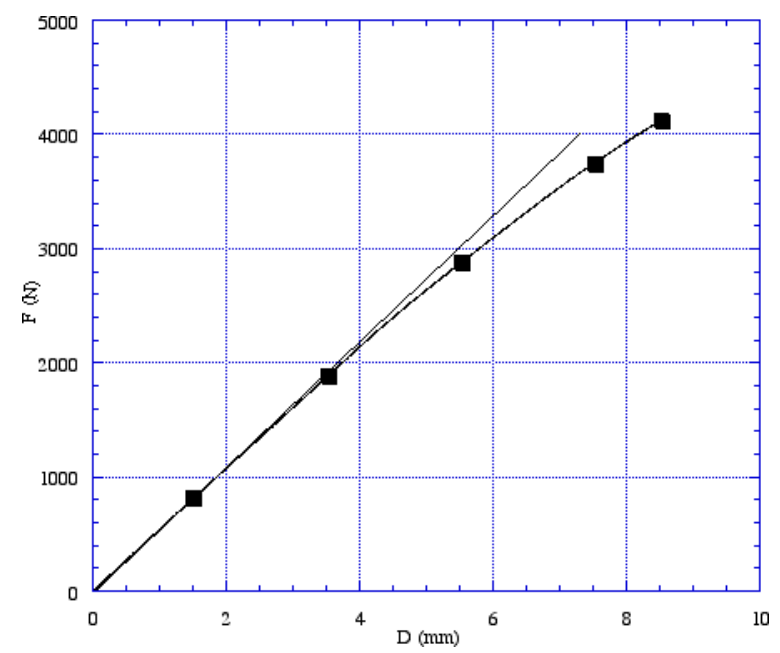

Figure 6. Courbe expérimentale force maximale $(F$, unité : $N)$ /déplacement maximum atteint $(D$, unité : $m m)$ aux points $\left(B_{i=1, \ldots, 5}\right)$ (figure 5$)$

ture de fibre (déformation longitudinale locale/déformation longitudinale maximale admissible), est également calculé.

Expérimentalement, la valeur de la force pour laquelle la chute de la pente (sécante) de la courbe force/flèche devient significative (chute voisine de $2 \%$ ) est de $2500 \mathrm{~N}$ (figure 6). Pour cette valeur, le déplacement du couteau est voisin de 4,5 mm.

Numériquement, pour cette valeur d'effort, on relève un déplacement moyen voisin de 4,5 mm vers le bas des nœuds de la peau inférieure, opposés à ceux chargés. Le critère de rupture est voisin de 1 dans les éléments médians de la peau inférieure situés le long de l'axe $\vec{y}$, et très largement supérieur à 1 dans les éléments opposés de la peau supérieure, c'est-à-dire ceux auxquels appartiennent les nœuds sollicités. Le comportement utilisé (élastique linéaire) ne permet pas de modéliser les zones de plasticité locale qui peuvent exister sous le couteau. C'est la raison pour laquelle les valeurs du critère sont si élevées à cet endroit. Néanmoins, cette différence entre la valeur du critère calculée sur la peau inférieure et celle calculée sur la peau supérieure reflète le fait que sous le couteau, les contraintes et les déformations sont réellement bien plus élevées que sur la peau inférieure (où les états de contraintes et de déformation tendent vers un état uniaxial). Ce fait est confirmé expérimentalement : on observe très nettement que la rupture de l'éprouvette s'amorce sous le couteau pour se propager vers la peau inférieure.

On peut donc conclure que pour une valeur du déplacement du couteau voisine de $4,5 \mathrm{~mm}$, le phénomène de rupture des fibres, sur la peau inférieure, est au voisinage de son initiation, mais a déjà significativement débuté. 


\subsection{Analyse des mesures micro-ondes et mise en corrélation avec le phénomène de rupture des fibres}

L'existence d'un champ de contrainte au sein d'un milieu influence ses propriétés diélectriques (Sandacci et al., 2005). Ici, le phénomène est mis en évidence par les mesures de l'amplitude du coefficient de réflexion pour l'éprouvette sous et hors charge (figure 7). Ainsi, dans le cas où des mesures faites sous charge mettent en évidence des variations, il n'est pas possible de distinguer, dans ces variations, quelle est la part due à l'existence du champ de contrainte, de celle véritablement induite par un autre phénomène, notamment l'endommagement. C'est la raison du second profil (charge/décharge, figure 5) d'application du déplacement du couteau. Plus précisément, son intérêt est d'abord de confirmer les mesures faites lors du chargement monotone jusqu'à rupture et ensuite, et surtout, de réaliser la mesure des grandeurs caractéristiques du circuit aux instants où le déplacement du couteau retrouve une valeur nulle.

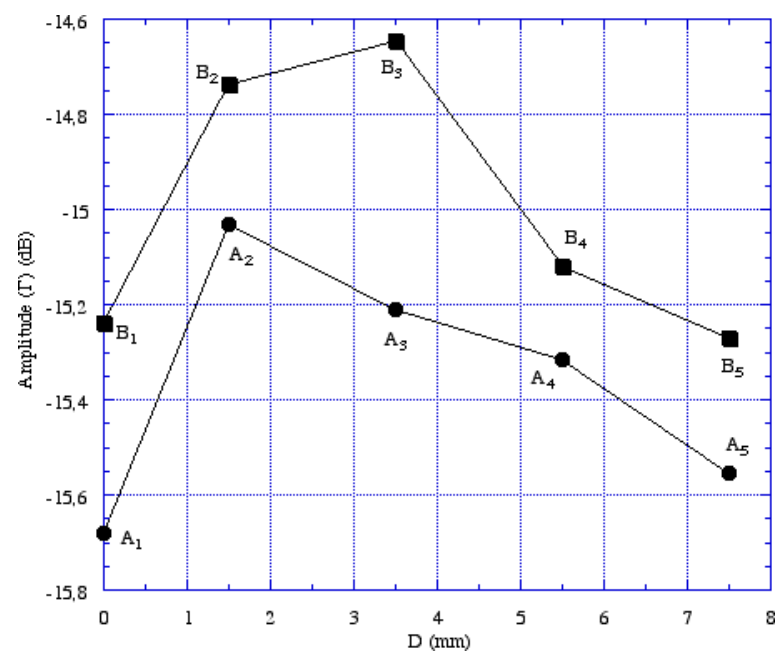

Figure 7. Amplitude du coefficient de réflexion (Amplitude ( $\Gamma$ ), unité : $d B$ ) en fonction du déplacement maximum atteint $(D$, unité : $m m)$ aux points $\left(A_{i=1, \ldots, 5}\right)$ sans charge et $\left(B_{i=1, \ldots, 5}\right)$ sous charge (figure 5) (Mesures à $2 \mathrm{GHz}$ ).

Dans la littérature, les méthodes de caractérisation diélectrique des matériaux à faible perte établissent que la variation de la partie imaginaire de $\Gamma$ est induite principalement par la variation de $\epsilon_{r}$. Parmi l'ensemble des grandeurs mesurées analysables, on va donc s'intéresser au coefficient $\frac{\Gamma-\Gamma_{R E F}}{\Gamma_{R E F}}\left(\Gamma_{R E F}\right.$ désigne le coefficient $\Gamma$ mesuré éprouvette non chargée à l'instant du retour à un déplacement nul après un déplacement maximum atteint de $1 \mathrm{~mm}$ ). A partir de l'analyse des mesures, on constate que cette grandeur montre des variations notables à partir d'un déplacement du couteau voisin de 4,5 mm (figure 8) Ce déplacement est en outre repéré expérimentalement 
(et par calcul par éléments finis) comme étant celui qui indique significativement le début de l'apparition des ruptures de fibres (figure 6). Egalement, en plus de l'absence de charge, aucun déplacement résiduel significatif n'est visible sauf après l'application d'un déplacement maximum supérieur à $8 \mathrm{~mm}$. Ainsi, en dessous de cette valeur, on peut affirmer qu'aucun autre phénomène dissipatif notable n'est présent au sein de l'éprouvette, autre que les ruptures de fibres. En conclusion, on peut affirmer que les variations de $\Gamma$ sont le reflet des variations de la permittivité relative du matériausubstrat dues à l'endommagement.

La méthode est donc bien capable de détecter la présence de l'endommagement.

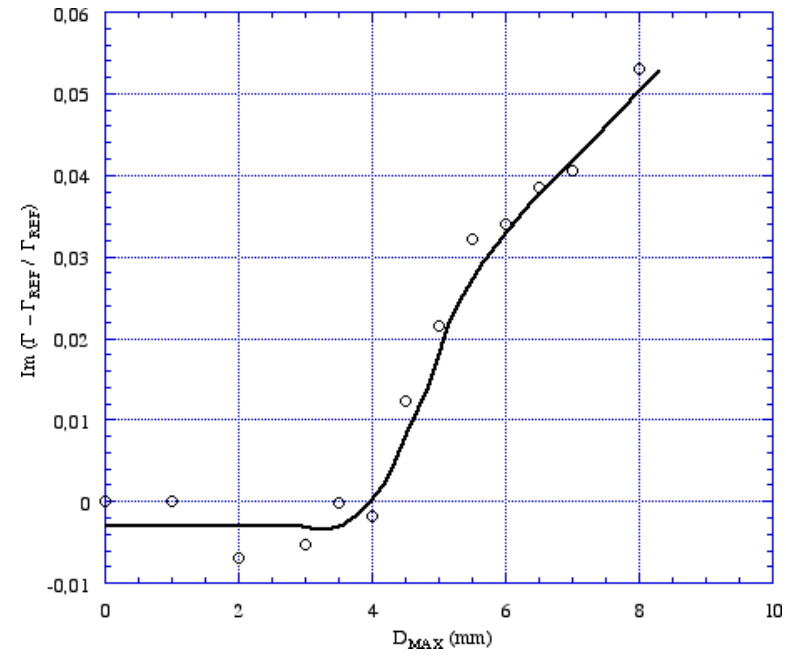

Figure 8. Partie imaginaire du coefficient $\frac{\Gamma-\Gamma_{R E F}}{\Gamma_{R E F}}$ (sans unité) en fonction du déplacement maximum atteint ( $D_{M A X}$, unité $: \mathrm{mm}$ ) (Issues de mesures aux points $\left(A_{i=1, \ldots, 5}\right)$ sans charge (figure 5$)$ ).

\section{D'autres intêrets de la méthode}

La méthode proposée présente potentiellement également les avantages suivants :

- une étude en profondeur suivant la fréquence de l'onde utilisée ;

- une détection plus ou moins locale, selon la géométrie du ruban;

- l'utilisation d'un ruban patchable permettant ainsi de réaliser de manière aisée des mesures à différents endroits de la structure à expertiser. 


\section{Conclusion}

Le travail présenté n'a pas l'ambition de répondre à toutes les interrogations légitimes qui sont associées au développement d'une nouvelle méthode de contrôle non destructif destinée à détecter des endommagements au sein des composites. Ici, dans un premier temps, on a seulement voulu montrer la faisabilité d'une méthode fondée sur l'observation et l'analyse des modifications des propriétés diélectriques d'un milieu composite dues à un endommagement. Dans un second temps, on a souhaité indiquer que cette méthode a potentiellement de sérieux atouts pour être une alternative intéressante et complémentaire aux techniques actuelles. Cette méthode obtient son diagnostic (la mise en évidence de l'existence d'un endommagement) via un circuit résonant microruban soumis à un champ électromagnétique dans la gamme des micro-ondes. La faisabilité de cette méthode a été démontrée dans le cadre d'expertise de matériaux composites unidirectionnels et plus précisément pour la détection de leur anisotropie et celle des ruptures de fibres dans une éprouvette en flexion. Ces premiers résultats, encourageants, doivent maintenant être complétés, notamment en se posant la question de savoir si cette méthode est non seulement capable de distinguer plusieurs endommagements en présence, mais aussi de les quantifier, notamment dans l'épaisseur du matériau.

\section{Bibliographie}

Abry J. C., Choi Y. K., Chateauminois A., Dalloz B., Giraud G., Salvia M., « In-situ monitoring of damage in CFRP laminates by means of AC and DC measurements », Composites Science and Technology, vol. 61, p. 855-864, 2001.

Akuthota B., Hughes D., Zoughi R., Myers J., Nanni A., « Near field microwave detection of disbond in carbon fiber reinforced polymer composites used for strengthening cement-based structures and disbond repair verification », Journal of materials in civil engineering, vol. 16, p. 540-546, 2004.

Aymerich F., Meili S., «Ultrasonic evaluation of matrix damage in impacted composite laminates », Composites Part B : Engineering, vol. 31, p. 1-6, 2000.

Balageas D., Levesque P., « EMIR : a photothermal tool for electromagnetic phenomena characterization », Revue Générale de Thermique, vol. 37, p. 725-739, 1998.

Ball R. J., Almond D. P., « The detection and measurement of impact damage in thick carbon fibre reinforced laminates by transient thermography », NDT \& E International, vol. 31, p. 165-173, 1998.

Blassiau S., Modélisation des phénomènes microstructuraux au sein d'un composite unidirectionnel carbone/époxy et prédiction de durée de vie : contrôle et qualification de réservoirs bobinés, Thèse de doctorat, Ecole des Mines de Paris, 2005.

Blassiau S., Thionnet A., Bunsell A., « Micromechanisms of load transfert in a unidirectional carbon-fibre epoxy composite due to fibre failures. Part 1 : Micromechanisms and 3D analysis of load transfert, the elastic case », Composite Structures, vol. 74, p. 303-318, 2006 a.

Blassiau S., Thionnet A., Bunsell A., « Micromechanisms of load transfert in a unidirectional carbon-fibre epoxy composite due to fibre failures. Part 2 : Influence of viscoelastic and 
plastic matrices on the mechanism of load transfert », Composite Structures, vol. 74, p. 319331, 2006b.

Bleay S. M., Humberstone L., « Mechanical and electrical assessment of hybrid composites containing hollow glass reinforcement », Composites Sciences and Technology, vol. 59, p. 1321-1329, 1999.

DeOliveira R., Frazão O., Santos J. L., Marques A. T., « Optic fibre sensor for real-time damage detection in smart composite », Computers \& Structures, vol. 82, p. 1315-1321, 2004.

Ganchev S. I., Runser R. J., Qaddoumi N., Ranu E., Carriveau G., « Microwave nondestructive evaluation of thick sandwich composites », Materials evaluation, vol. 53, p. 463-467, 1995.

Hammerstad E. O., « Equation for microstrip circuit design », Proceedings of the European Microwave Conference, Hamburg, Germany, p. 268-272, 1975.

Hill R., Brooks R., Kaloedes D., « Characterization of transverse failure in composites using acoustic emission », Ultrasonics, vol. 36, p. 517-523, 1998.

Huber C., Zoughi R., « Detecting stress and fatigue cracks », IEEE potentials, vol. 15, p. 20-24, 1996.

Hughes D., Behrens C., Zoughi R., Green G., Campbell P., « Microwave and millimeter wave inspection of impact in GFRP composite and pitted corrosion in steel », ASNT Spring Conference, Portland, OR., USA, March, 2002.

Kagawa Y., Zhu S., « Non-contact damage detection of fiber-reinforced ceramic matrix composites by electromagnetic wave », Ceramic transactions, vol. 128, p. 199-217, 2001.

LeGallou J. H., Ledieu M., Prigent J. C., Archer O., « New technique for microwave characterization of material under compressive stress », Conference on Precision Electromagnetic measurement, Ottawa, Canada, p. 16-17, June, 2002.

Mian A., Han X., Islam S., Newaz G., « Fatigue damage detection in graphite/epoxy composites using sonic infrared imaging technique », Composites Science and Technology, vol. 64, p. 657-666, 2004.

Minnaar K. I., Zhou M., « A novel technique for time-resolved detection and tracking of interfacial and matrix fracture in layered materials », Journal of the Mechanics and Physics of Solids, vol. 52, p. 2771-2799, 2004.

Okabe Y., Mizutani T., Yashiro S., Takeda N., « Detection of microscopic damages in composite laminates with embedded small-diameter fiber Bragg grating sensors », Composites Science and Technology, vol. 62, p. 951-958, 2002.

Pevzner P., Weller T., Berkovits A., « Use of heat emitted by broken optic fibers : A new approach for damage detection in composites », Engineering Failure Analysis, vol. 12, p. 860874, 2005.

Pliquet B., Levesque P., Duchêne B., Ferrieres X., Alliot J. C., « Electromagnetic nondestructive evaluation of materials from incomplete EMIR data », 26th Quantitative Nondestructive Evaluation Conference, Montreal, Canada, July, 1999.

Rippert L., Wevers M., VanHuffel S., « Optical and acoustic damage detection in laminated CFRP composite materials », Composites Science and Technology, vol. 60, p. 2713-2724, 2000.

Sandacci S., Makhnovskiy D., Panina L., Larin V., « Stress-Dependent Magnetoimpedance in Co-Based Amorphous Wires with induced axial anisotropy for Tunable Microwave Composites », IEEE Trans. on magnetics, vol. 41, p. 3553-3555, 2005. 
278 Revue des composites et des matériaux avancés. Volume $16-\mathrm{n}^{\circ}$ 3/2006

Scarponi C., Briotti G., «Ultrasonic technique for the evaluation of delaminations on CFRP, GFRP, KFRP composite materials », Composites Part B : Engineering, vol. 31, p. 237-243, 2000.

Schilling P. J., Karedla B. R., Tatiparthi A. K., Verges M. A., Herrington P. D., « X-ray computed microtomography of internal damage in fiber reinforced polymer matrix composites », Composites Science and Technology, vol. 65, p. 2071-2078, 2005.

Schueler R., Joshi S. P., Schulte K., « Damage detection in CFRP by electrical conductivity mapping », Composites Science and Technology, vol. 61, p. 921-930, 2001.

Shrestha S., Kharkovsky S., Zoughi R., Hepburn F., « Microwave and millimeter wave nondestructive testing of the space shuttle external tank insulating foam », Materials evaluation, vol. 63, p. 339-344, 2005.

Surgeon M., Wevers M., « Modal analysis of acoustic emission signals from CFRP laminates », NDT \& E International, vol. 32, p. 311-322, 1999.

Wevers M., Rippert L., Papy J. M., VanHuffel S., « Processing of transient signals from damage in CFRP composite materials monitored with embedded intensity-modulated fiber optic sensors », NDT \& E International, vol. 39, p. 229-235, 2006.

Xu Y., Leung C. K. Y., Tong P., Yi J., Lee S. K. L., « Interfacial debonding detection in bonded repair with a fiber optical interferometric sensor », Composites Science and Technology, vol. 65, p. 1428-1435, 2005.

Yan Y. J., Yam L. H., « Online detection of crack damage in composite plates using embedded piezoelectric actuators/sensors and wavelet analysis », Composite Structures, vol. 58, p. 2938, 2002. 